\title{
OPEN Preclinical therapeutics ex ovo quail eggs as a biomimetic automation-ready xenograft platform
}

\author{
Samuel V. Rasmussen ${ }^{1}$, Noah E. Berlow ${ }^{1}$, Lisa Hudson Price ${ }^{1}$, Atiya Mansoor ${ }^{1}$, \\ Stefano Cairo ${ }^{1,2}$, Sandra Rugonyi ${ }^{3}$ \& Charles Keller ${ }^{1 \bowtie}$
}

Preclinical cancer research ranges from in vitro studies that are inexpensive and not necessarily reflective of the tumor microenvironment to mouse studies that are better models but prohibitively expensive at scale. Chorioallantoic membrane (CAM) assays utilizing Japanese quail (Coturnix japonica) are a cost-effective screening method to precede and minimize the scope of murine studies for anti-cancer efficacy and drug toxicity. To increase the throughput of CAM assays we have built and optimized an 11-day platform for processing up to 200 quail eggs per screening to evaluate drug efficacy and drug toxicity caused by a therapeutic. We demonstrate ex ovo concordance with murine in vivo studies, even when the in vitro and in vivo studies diverge, suggesting a role for this quail shellfree CAM xenograft assay in the validation of new anti-cancer agents.

Pediatric cancer has historically limited new drug development as demonstrated by only 10 new agents earning primary childhood cancer FDA approval since 1978. To develop new therapies for rare disease, a cost-effective workflow from basic science target identification to preclinical research and then to clinical investigation is needed. Current preclinical research approaches move from in vitro studies to in vivo murine models; however, most of the time drug response data obtained from in vitro assays fail to be confirmed in vivo. As mouse studies are sometimes prohibitively expensive and time-consuming, taking tens of thousands of dollars and 10 or more weeks to complete, it is crucial to develop innovative cost and time-effective processes to improve the selection of anti-cancer agents to be prioritized for preclinical mouse studies Here we propose a re-examined and optimized the shell-free quail chorioallantoic membrane assay (CAM) as a precursor to mouse preclinical studies ${ }^{1,2}$.

CAM assays have traditionally utilized chicken (chick) or quail eggs, with chick being used more commonly. Chick CAM models have been employed for the study of angiogenesis, tumor growth and metastasis ${ }^{3-5}$. The CAM is a membrane formed by the fusion of the chorion and allantois membrane on embryonic day 5-6 (e5-6) ${ }^{6}$. The membrane will attach to the inside of the eggshell allowing respiration and calcium extraction for the growing embryo. The methods for culturing embryos fall into two categories: in ovo, whereby a small hole cut into the shell gives access to the embryo (for example, the studies performed by Sidney Farber in $1962^{5}$ ), or ex ovo with the embryo transferred to a cell culture plate and grown separate from the shell. The chick methods of in ovo incubation, while effective for chick survival, are time consuming and cannot be scaled because of the need for a skilled operator ${ }^{7}$. For ex ovo approach, another group has reported a culture method for Japanese quail that is novel but also time intensive and unsuitable for automation in multi-well plates ${ }^{4,8}$. Parenthetically, too, the Japanese quail (Coturnix japonica) genome was sequenced in 2016 and is publicly available.

Approaches to using the avian eggs and/or CAM assays have had preclinical drug administration (dosing) pharmacokinetic challenges, but in recent years the application of drugs into the quail or chick has been approached by topical and intravenous injection ${ }^{9}$. To circumvent systemic drug administration, drug can be admixed with tumor cells in an extracellular matrix that is applied to the quail CAM ${ }^{1,3}$.

CAM experiments have been conducted on a variety of cancers, but to our knowledge no medium- or highthroughput methods have been reported for either adult or pediatric cancers. Herein, we present an approach to increased throughput of CAM assays in the preclinical prioritization of anti-cancer compounds via an automation-ready 6-well plate format.

${ }^{1}$ Children's Cancer Therapy Development Institute, 12655 SW Beaverdam Rd West, Beaverton, OR 97005, USA. ${ }^{2}$ Xentech, Evry, France. ${ }^{3}$ Department of Biomedical Engineering, Oregon Health and Science University, Portland, OR 97239, USA. ${ }^{\varpi}$ email: charles@cc-tdi.org 


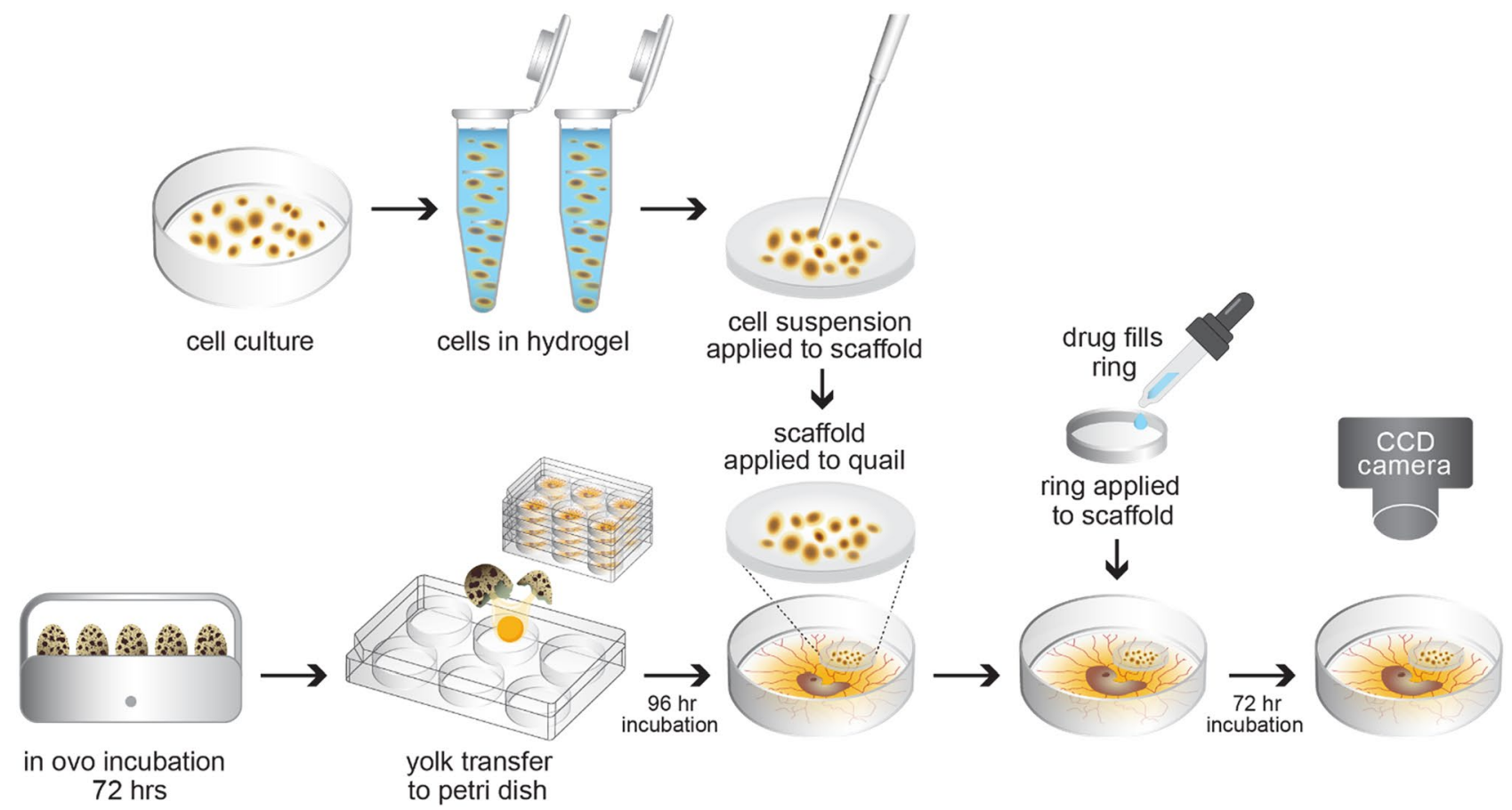

Figure 1. Schematic for the quail egg drug screening assay. The quail eggs are incubated for $72 \mathrm{~h}$ and then the embryos are transferred to six-well plates. The six-well plates are incubated for $96 \mathrm{~h}$ then any non-viable egg contents are removed. During the quail egg incubation, tumor cells are cultured. After the $96 \mathrm{~h}$ incubation the tumor cells are suspended in hydrogel, added to a 3D scaffold, and applied to the chorioallantoic membrane. A $9 \mathrm{~mm}$ ring is placed onto the 3D scaffold and then filled with P-10 osmotic beads soaked and equilibrated in the therapeutic compound. The quail is then incubated for $72 \mathrm{~h}$. To analyze drug efficacy on tumor cells, luciferin is added to the scaffold and then imaged by a CCD camera to determine the total xenograft cell viability. Copyright holder Children's Cancer Therapy Development Institute.

\section{Results}

Egg cracking and viability. A schematic of the shell-free quail CAM assay is presented in Fig. 1. A ratelimiting step is the transfer of egg contents with the yolk intact to a multi-well plate. A simple apparatus to process 6 eggs at a time is shown in Fig. $2 \mathrm{a}-\mathrm{c}$. The total time required to conduct the transfer from in ovo to ex ovo for 100 eggs is approximately $2 \mathrm{~h}$ and $10 \mathrm{~min}$. This approach has intact-yolk transfer rate of $85 \%$ on average as shown in Fig. 2d, with egg content transfer to multi-well plates is consistently achieved as shown in Fig. 2e. A second challenge was to determine the stage of egg development with the least spontaneous embryo death. From our studies, embryo survival decreases to $40-50 \%$ survival by embryonic day 10 with most of the losses between embryonic day 4 and day 7 (Fig. 2f); therefore, day 7 (e7) (Fig. 3a) was identified as the best timepoint for tumor xenografting.

Validation of the reporter systems used. We next confirmed bioluminescence of tumor cells using a commercially-available luciferase-red fluorescent protein (RFP) lentivirus reporter system (Supplementary Fig. 1). The luminescence was linear with the tumor cell counts of xenograft-ready "modules" consisting of tumor cells embedded in hydrogel on a fiberglass scaffold (Supplementary Fig. 1). As a cautionary note, for the HepG2 cell line the luminescence and cell number were linear in our range employed but became non-linear at $4 \times 10^{6}$ cells per tumor module (Supplementary Fig. 1). A transgenic rhabdomyosarcoma cell culture (U484842) demonstrated a linear relationship between luminescence and tumor cell number for the range of $1 \times 10^{5}$ and $1.25 \times 10^{6}$ cells per module (data not shown). These ranges can be considered narrow however they provide an accurate linear range to compare and are bright enough to ensure visualization by bioluminescence.

Ex ovo efficacy testing at single drug concentrations. We initially validated our system using the dual PI3K/mTOR inhibitor BEZ235 was tested on the rhabdomyosarcoma cell culture U484842. A 3D scaffold (Fig. 3b) was used for the tumor module which was supported by the capillary-rich CAM (Fig. 3c,d). We tested BEZ235 at $500 \mathrm{nM}$ in the hydrogel volume (Fig. 3e) and had an observable $20 \%$ reduction in U48484 tumor module bioluminescence (tumor cell viability) as shown in Fig. 3f. We are cautious to note, however, that the efficacy of BEZ235 could have been decreased by diffusion into the CAM, which led us to pursue a drug depot/ osmotic drug release approach described below.

In vitro and ex ovo efficacy testing across a drug concentration range. A PLK inhibitor (volasertib) was selected for testing against a range of hepatoblastoma cell lines given that PLK1 is a proposed thera- 
a

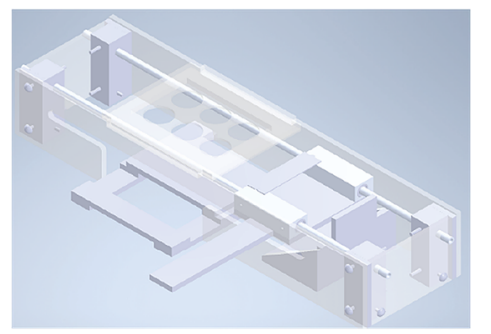

c

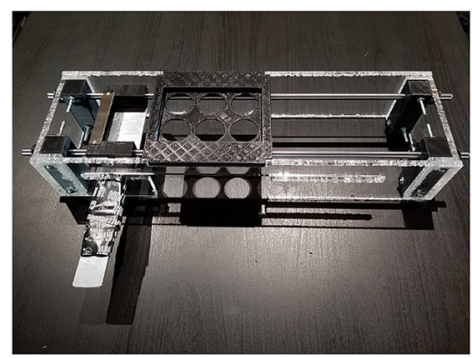

e

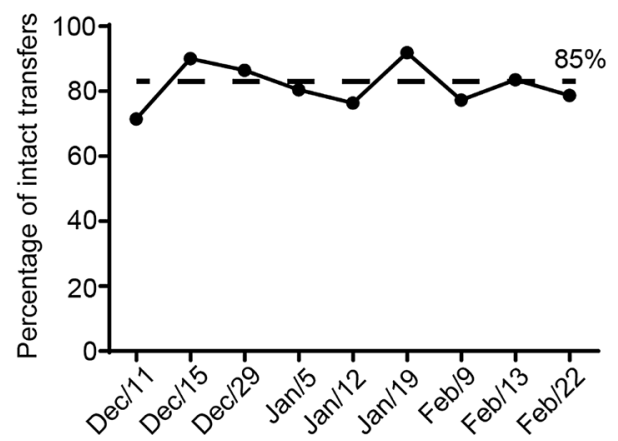

b

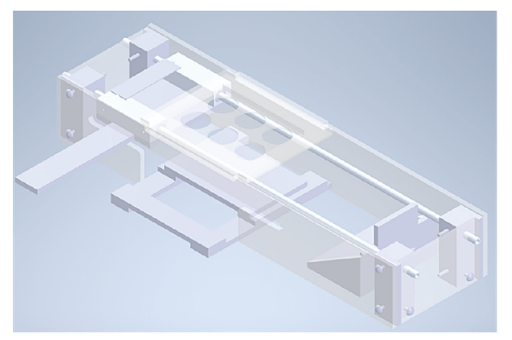

d

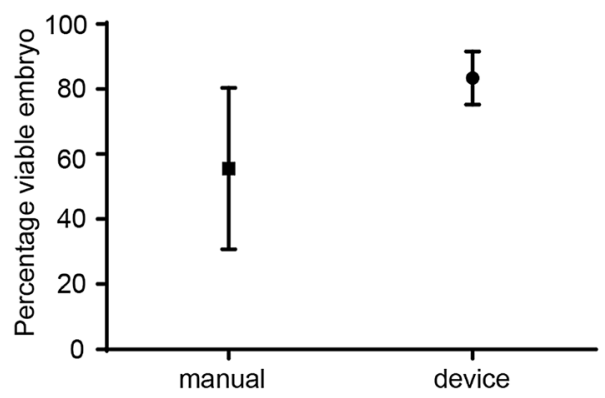

f

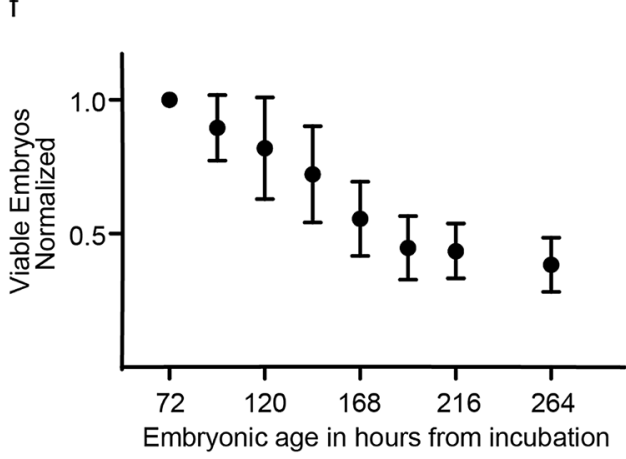

Figure 2. Embryo Transfer to multi-well plates. (a,b) CAD drawing of the quail egg opener in the before and after positions for opening quail eggs. (c) The blade collision device to open the quail eggs and transfer them to the six-well plate. (d) The quail batches used for the device method varied between 40 and 75 quail eggs per batch with 8 batches done. Three batches were used for the manual forceps method had between 32 and 75 quail eggs each with 3 batches done, error bars are standard deviation. (e) Number of viable egg yolks transferred to six-well plates weekly over a period of 9 weeks. (f) The age vs survival of the quail embryos used to choose the experiment start date error bars are standard deviation.

peutic target ${ }^{10}$. The canonical hepatoblastoma cell line HepG2 and patient-derived xenograft (PDX) explanted cell lines HB243 and HB282 were selected as contemporary, robust patient-derived comparators ${ }^{11}$. Volasertib was tested both in vitro and ex ovo against the cell lines across a concentration range. Previous in vitro studies ${ }^{10}$

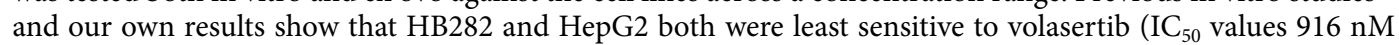
Fig. 4a and $508 \mathrm{nM}$ in Fig. 4b, respectively), whereas HB243 is considered the most sensitive (IC ${ }_{50} 191 \mathrm{nM}$ in Fig. 4a). For CAM xenografts of these cell cultures, drug delivery is different than in vitro because of CAM vessel washout and embryo drug metabolism; however, in these experiments drug exposure to the engraftment modules was kept constant by osmotic bead drug delivery. Given the small blood volumes of the quail (Supplementary Fig. 2) and the desire to automate drug testing, the osmotic bead drug delivery system was chosen and validated for consistent small-molecular research (Supplemental Fig. 3) as the most cost-effective method to have a defined drug exposure/drug delivery over time. Ex ovo, HB243 and HB282 were congruent to in vitro studies with a $50 \%$ tumor growth inhibition (ex ovo $\mathrm{IC}_{50}$ ) of $1455 \mathrm{nM}$ and $1040 \mathrm{nM}$, respectively (Fig. $4 \mathrm{c}$ ). On the other hand, HepG2 was insensitive at any concentration as shown in Fig. $4 \mathrm{~d}$ in stark contrast to the in vitro studies.

Murine in vivo xenograft study comparisons. In cell line-based mouse xenograft studies, HB243 and HepG2 had different responses to volasertib in vivo. HB243 caused a statistically significant reduction in growth relative to the control group as shown in Fig. 4e with the treatment mice surviving to the endpoint an average of 4.5 days longer than control (vehicle). HepG2 had no increase in survival in Fig. $4 \mathrm{f}$ with no significant difference between the volasertib-treated cohort and the control cohort. Volasertib resistance in HepG2 could not be attributed to PLK expression or ABC transporter (drug efflux) expression (Supplementary Table 1) as we did not find a clear pattern with ABC genes in HepG2 having a consistently higher expression than HB243 and HB282 however some such as ANCG2 were expressed more in HepG2. 
a

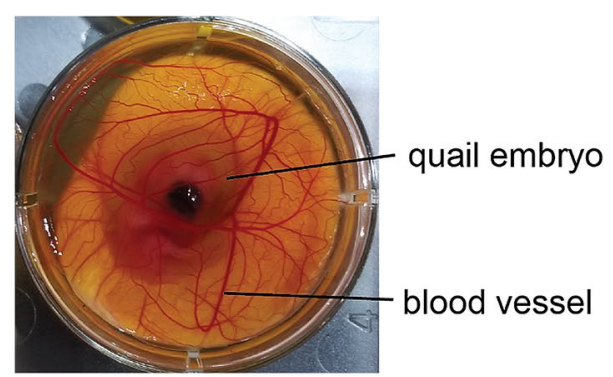

C

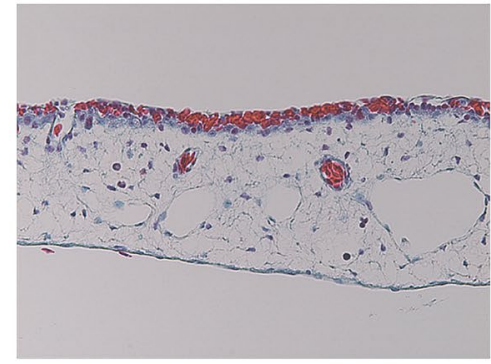

e

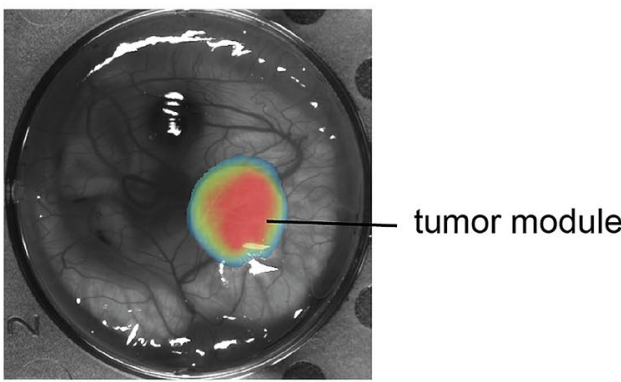

b

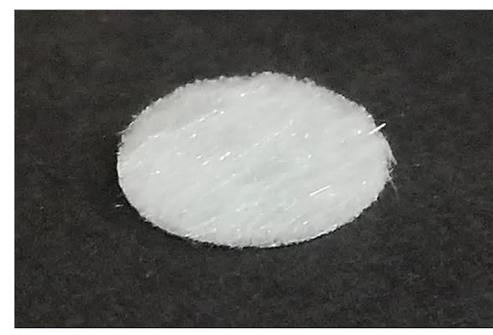

d

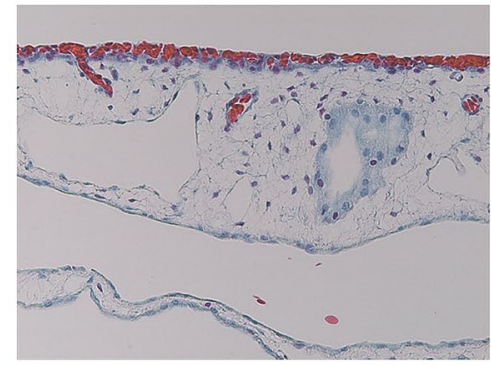

f

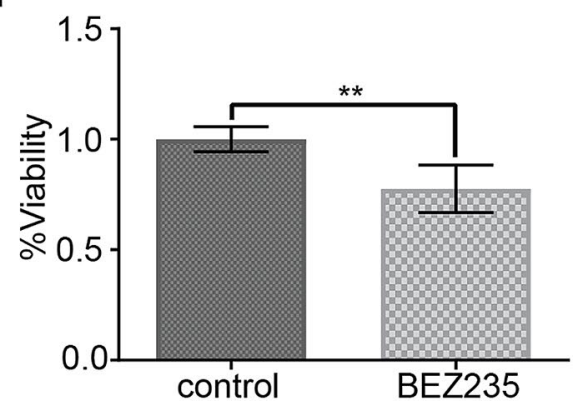

Figure 3. Quail xenograft assay. (a) a quail embryo at e7. (b) An unused 3D scaffold $9.5 \mathrm{~mm}$ in diameter for use as a tumor module. (c,d) Cross sections of the CAM. (e) representative pseudo-colorized image of viable, luciferase-expressing tumor cells $72 \mathrm{~h}$ after xenografting on the CAM. (f) The assay used murine rhabdomyosarcoma cells treated with BEZ235 $(n=6)$ or vehicle control $(n=4)$. ${ }^{* *}$, significance $p=0.0048$.

Ex ovo patient-derived xenografts. Because generation of patient-derived xenograft using immunecompromised host mice can take 2-7 or more months ${ }^{12}$, we tested whether flat sections of patient tumor would engraft on the CAM immune-tolerant platform (Supplementary Fig. 3). Tumor engraftment (vascularization) from a mouse PDX-derived explant from an Ewing sarcoma occurred within $24 \mathrm{~h}$ and viability was maintained for $96 \mathrm{~h}$ (Supplementary Fig. 3). Similar results were obtained with an autopsy-derived Ewing sarcoma specimen cryo-preserved, thawed, and engrafted on the CAM (Supplementary Fig. 3).

Quail toxicity assay. The histology slides were examined by co-pathologist A.M and were found to have significantly more toxicity from the combination of cediranib and erlotinib than either drug alone. Synergistic toxicity was seen in the e10 quail embryo liver and kidney as shown in Fig. 5.

\section{Discussion}

Herein we report methods for reliably and reproducibly performing ex ovo drug testing in shell-free quail CAM assay xenografts. We believe this platform brings us close to automation through mechanical systems for transferring egg contents to multi-well plates, and we demonstrate the feasibility of single concentration xenograft testing, or xenograft testing across a concentration range (e.g., the ex ovo $\mathrm{IC}_{50}$ ). When culturing the quail ex ovo we noticed a majority of the die off between e4 and e7 most likely due to the shock of transferring the embryo to the six well plate. At e7 the chorioallantoic membrane had fully formed and providing a capillary rich surface to support the tumor module. We tested volasertib in mouse xenografts and compared the in vivo results to the ex ovo quail xenograft results and observed that HepG2 continued to be resistant in concordance with the ex ovo experiments and in contrast to the in vitro experiments. HB243 had a statistically significant reduction in growth in concordance to both the sensitivity of HB243 to volasertib in the ex ovo and in vitro experiments. The resistance of HepG2 to volasertib in vivo and ex ovo but not in vitro deserves further analysis. We conducted RNA 

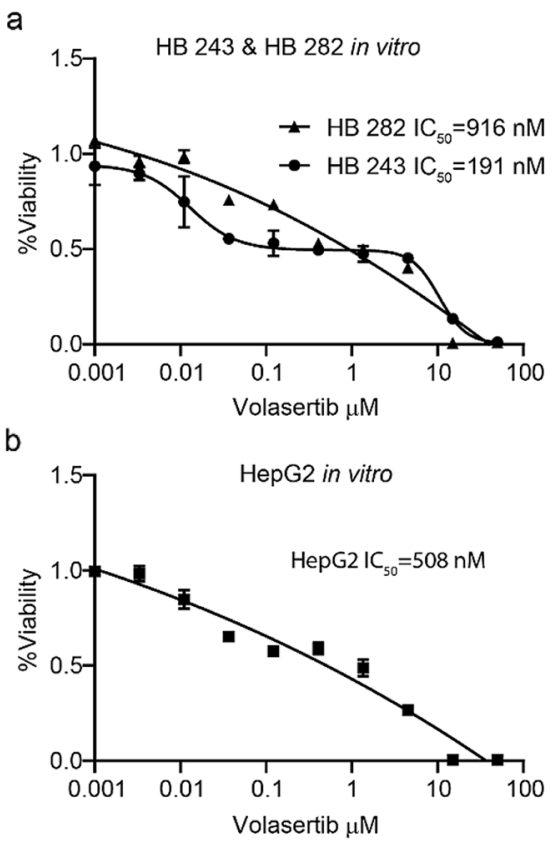

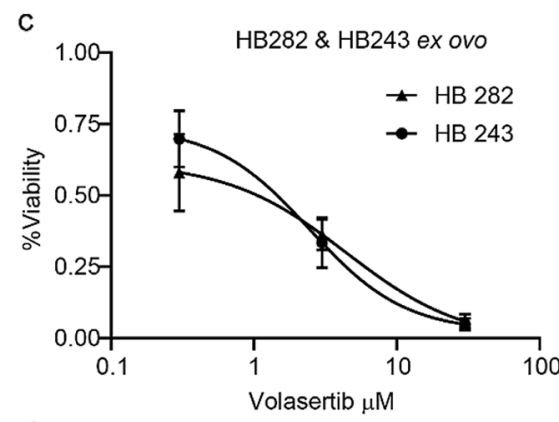

d

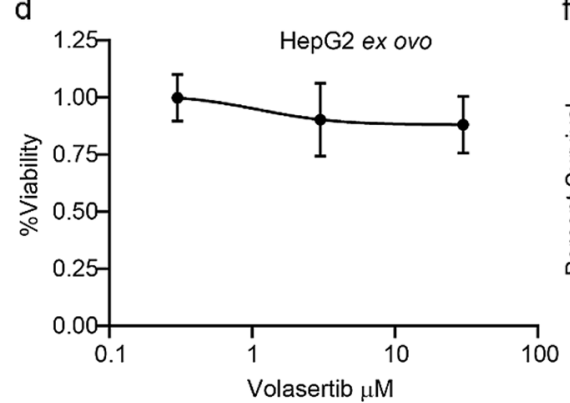

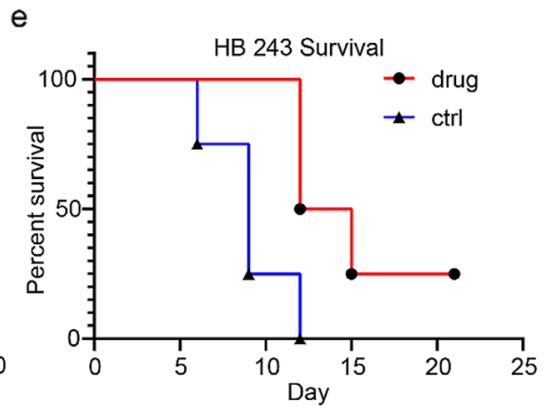

f

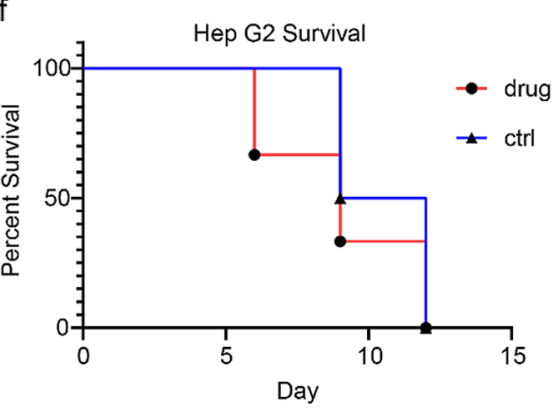

g

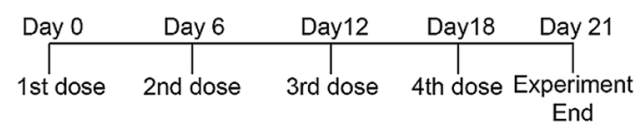

Figure 4. Comparison of in vitro, quail egg ex ovo xenograft and mouse in vivo xenograft results. (a,b) In vitro drug curves, each point has $n=4$ replicates. (c,d) For the ex ovo drug curves, each concentration has $n=4-6$ replicates. (e) The murine xenograft survival analyses for HB243 $(n=4)$ defined an event occurrence as tumor volume equal or greater than $0.75 \mathrm{cc}$, and a significant difference observed between treatment and control groups with a $\mathrm{p}=0.031 \mathrm{using}$ the Mantel-Cox test. (f) The survival of HepG2 bearing mice $(\mathrm{n}=3)$ using the same criteria did not have a difference between treatment and control groups with $p=0.685$. (g) Shown is the experiment timeline for drug dosing in mouse xenograft studies.
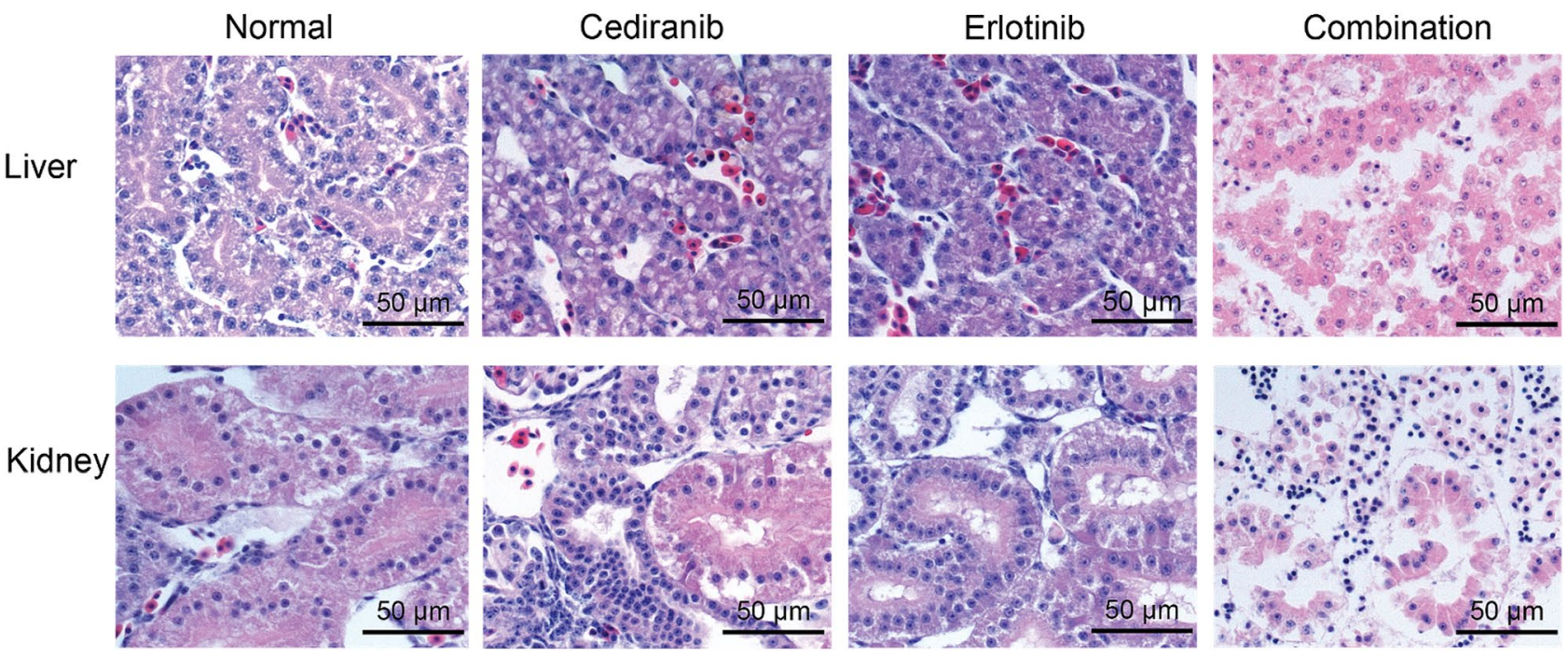

Figure 5. Histology slides stained with $\mathrm{H} \& \mathrm{E}$ of embryonic quail. Comparison of liver and kidney for e10 quail dosed with the human clinical $\mathrm{C}_{\max }$ of cediranib (VEGFR and poly-kinase inhibitor) and/or erlotinib (EGFR inhibitor) revealing synergistic toxicity in liver and kidney.

sequencing that could not discern $\mathrm{ABC}$ transporters overexpression as the cause of resistance. Our validation studies were conducted solely for hepatoblastoma, but parallel remain to be done for other pediatric and adult cancers. The readiness in which patient xenografts engraft to the CAM is an exciting opportunity for further research. 
Future directions will include addressing the pharmacokinetic considerations for efficacy and toxicity models in lieu of our described approach of hydrogel-based tumor modules as a single compartment model. Given the small blood volumes of the quail CAM, and the changing blood volumes from e7 to e11, serial micro-sampling approaches will require careful but worthwhile optimizations.

\section{Methods}

Quail preparation. All experiments were conducted in accordance with Children's Cancer Therapy Development Institute policies and all relevant guidelines. Coturnix japonica eggs were purchased from Boyd's Bird Company (Eagle Creek, OR) and PurelyPoultry (Fremont, WI), stored at $4{ }^{\circ} \mathrm{C}$ for $120 \mathrm{~h}$ and then incubated at $37^{\circ} \mathrm{C}$ and $70 \%$ humidity for approximately $72 \mathrm{~h}$. Quail eggs were opened by our mechanical device (Fig. 2a) and the embryos and white transferred to a six-well plate. Six-well plates were incubated for $96 \mathrm{~h}$ (embryonic day 7 , e7) at which point unfertilized/non-viable egg contents were removed and viable quail used for assays.

Cell lines. All cell lines were obtained as de-identified samples. HepG2 human liver carcinoma cells (ATCC, HB-8065) were transfected with lentiviral particles containing RFP, luciferase, and neomycin resistance following manufacturer's instructions (cat\#LVP677, Gentarget, San Diego, CA). Reporter-transfected cells were purified using $800 \mathrm{nM} \mathrm{G} 418$ antibiotic selection $24 \mathrm{~h}$, flow sorted, then antibiotic selected again with the resulting cell line stably expressing RFP and luciferase. Cells were maintained in DMEM (cat\#11990573, ThermoFisher Scientific, Waltham, MA) with 10\% FBS (ThermoFisher Scientific, cat\#10437036) and 1\% penicillin-streptomycin (cat\#15140122, ThermoFisher Scientific). HB282 was received from co-author Stefano Cairo [Xentech] and transfected with a lentiviral particle containing RFP, luciferase, and puromycin resistance following manufacturer's instructions (cat\#LVP674, Gentarget). HB282 followed the previously listed selection process but with a puromycin (cat\#73342, Stemcell Technologies, Cambridge, MA) concentration of $2 \mu \mathrm{g} / \mathrm{ml}$. HB282 was cultured in ADMEM, 10\% FBS, $1 \%$ penicillin-streptomycin, 1\% L-glutamine. We received HB243 transfected with GFP and luciferase from co-author Stefano Cairo[Xentech]. Previously characterized U48484 murine alveolar rhabdomyosarcoma (aRMS) cells which stably express a luciferase reporter transgene were maintained in DMEM, $10 \%$ FBS, and $1 \%$ penicillin-streptomycin ${ }^{2}$.

Luminescence calibration. All experiments were conducted in accordance with Children's Cancer Therapy Development Institute policies and all relevant guidelines. To generate a standard curve for luminescence, 7 different cell densities ranging from 0 to $4 \times 10^{6}$ of HepG2Glo were suspended in Hydrogel-c (cat\#GS313, ESI-BIO, Alameda, CA) and $50 \mu \mathrm{l}$ were added to $9.5 \mathrm{~mm}$ diameter sterilized fiberglass 3D mesh in Supplementary Fig. 1 (cat\# SC-S510-0001, LenaBioscience, Atlanta, GA) according to manufacturer's protocol in a 24 well plate. Two hundred $\mu \mathrm{l}$ of luciferin-d (cat\#122,799, PerkinElmer, Waltham, MA) at $15 \mathrm{mg} / \mathrm{ml}$ diluted in PBS was added to each well, incubated for $10 \mathrm{~min}$, and then imaged using a UVP Biospectrum 600 (Analytik JenaUS LLC, Upland, CA). Luminescence readings were processed using Prism 8.0 (Graphpad Software, San Diego, CA, https://www.graphpad.com/scientific-software/prism/). Luminescence calibration was performed in the same manner for U48484 mouse aRMS cells with the range of cells from 0 to $1.5 \times 10^{6}$ cells per module.

Patient-derived xenograft onto the CAM. A diagram of the procedure is presented in Supplementary Fig. 3 with all experiments carried out performed after receiving approval from the institutional animal care and use committee (IACUC) at Children's Cancer Therapy Development Institute. Samples were collected from patients who had given informed consent and enrolled in the CuReFAST tumor banking study approved of by the Children's Cancer Therapy Development Institute's Institutional Review Board (Advarra, protocol \# cc-TDIIRB-1). An Ewing sarcoma patient derived xenograft from a 23 year-old male surgically implanted to a NSG mouse at Jax laboratory (model ID TM01617) was removed from the mouse, encased in $2 \%$ agarose at $37^{\circ} \mathrm{C}$ and sliced to $1 \mathrm{~mm}$ thick. The tumor slices were removed from the agarose and applied to an injury site on e7 quail embryos. Injury was created by placing a dry glass rod against the cam and carefully removing the glass rod. Twenty $\mu$ l of Matrigel was added on top of the tumor slice to covering the slice to avoid any drying. The tumor engrafted for $96 \mathrm{~h}$ and was then removed and fixed in formalin. Samples were then sectioned and $\mathrm{H} \& \mathrm{E}$ stained at Oregon Health \& Science University (OHSU) Histopathology Shared Resource.

Chick to quail blood volume comparison. All experiments were conducted in accordance with Children's Cancer Therapy Development Institute policies and all relevant guidelines. In order to develop pharmacokinetic approximations for the quail we compared literature sources for chicken embryo mass and blood volume growth to our measured quail embryo growth over the same Hamburger and Hamilton stages Supplementary Fig. 4 . We assumed that the ratio of blood volume to body mass would be the same during the same growth stages.

Quail tumor assay. All experiments were conducted in accordance with Children's Cancer Therapy Development Institute policies and all relevant guidelines. U48484 mouse rhabdomyosarcoma cells were cultured, trypsinized and added to two different vials of hydrogel making a concentration of $10^{6}$ cells per $50 \mu$. BEZ235 (cat\#S1009, Selleck Chemicals, Houston, TX) in a solution of 0.1\% DMF (cat\#TS-20673, Thermo Fisher Scientific) in PBS was added to $10^{6} \mathrm{U} 48484$ mouse rhabdomyosarcoma cells mixed with $50 \mu$ hydrogel for a final concentration of $500 \mathrm{nM}$ BEZ235. For untreated eggs, 0.1\% DMF in PBS was used as a control. $50 \mu \mathrm{l}$ of cells/ hydrogel/drug mixture were added to each scaffold and incubated for approximately $30-45 \mathrm{~min}$ at $37^{\circ} \mathrm{C}$ and $100 \%$ humidity. As detailed above, a superficial injury was created on the chorioallantoic membrane and tumor 
module containing either drug or control was placed on top. Quail bearing tumor module models were incubated for $72 \mathrm{~h}$. Add the end of the incubation, $100 \mu \mathrm{l}$ of PBS containing $1.5 \mathrm{mg}$ of luciferin-d (cat\#122,799, PerkinElmer) was added to the 3D scaffold, incubated for $10 \mathrm{~min}$ in the dark, and bioluminescence was measured using a Fluorchem instrument (ProteinSimple, San Jose, CA). The quail were imaged with an 8 min exposure for total light emission.

Quail dose response assay. All experiments were conducted in accordance with Children's Cancer Therapy Development Institute policies and all relevant guidelines. The tumor modules for dose response assay were generated as described above but with $5 \times 10^{5}$ cells per $50 \mu \mathrm{l}$. Drug was dissolved in DMSO for all levels to a final tumor module concentration of vehicle control, $0.3 \mu \mathrm{M}, 3 \mu \mathrm{M}$, or $30 \mu \mathrm{M}$ with $\mathrm{n}=6$. P-10 beads (cat\#1,504,144, Bio-Rad, Hercules, CA) were soaked in PBS at the concentration of the modules for four hours at room temperature. Approximately $50 \mu \mathrm{l}$ of bead solution was added to a $9.5 \times 1.5 \mathrm{~mm}$ plastic ring placed on top of the tumor module forming a drug depot. The drug depot provided a constant source of drug keeping the tumor module at a constant concentration despite drug leaving the module for the quail, as shown in Supplementary Fig. 2. The IR820 had an exponential range between 0.001 and $1 \mu \mathrm{M}$ as shown in Supplementary Fig. 2. The IR820 diffused through a tumor module at a constant rate and less than $10 \%$ of the IR820 diffused through as shown in Supplementary Fig. 2. The quail with tumor module models and drug depots were incubated for $72 \mathrm{~h}$. Afterwards, $150 \mu \mathrm{l}$ of $15 \mathrm{mg} / \mathrm{ml}$ luciferin (cat\#122,799, Perkin Elmer) in PBS was added to the modules incubated for $10 \mathrm{~min}$ and then imaged on an IVIS Lumina (PerkinElmer) for between $15 \mathrm{~s}$ (for cell lines HepG2) or $1 \mathrm{~min}$ (for cell lines HB243 and HB282).

In vivo mice experiment. All studies in mice were performed after receiving approval from the institutional animal care and use committee (IACUC) at Children's Cancer Therapy Development Institute and in accordance with ARRIVE Guidelines. Hep G2 and HB243 were suspended in Matrigel and injected into $\mathrm{n}=10$ eight week-old female nod scid gamma mice per cell line xenograft (Charles River, Hollister, CA, NOD.CB17$\mathrm{Prkdc}^{\text {scid }} / \mathrm{NCrCrl}$ ) with $2 \times 10^{6}$ cells per $100 \mu \mathrm{l}$ injection. The dosing schedule shown in Fig. $4 \mathrm{~g}$ began after reaching 0.25 cubic centimeters in volume with mice that did not develop tumors excluded. Volasertib (cat\# S2235, SelleckChem) was suspended in 3.75\% DMSO and corn oil (cat\# C8267, Sigma-Aldrich, St Louis, MO) at a concentration of $1.5 \mathrm{mg} / \mathrm{ml}$ with $100 \mu \mathrm{l}$ injected intraperitoneally with mice chosen for the drug and control group randomly selected to minimize selection bias. The mice were imaged by intraperitoneal injection of luciferin-d according to manufacturer's instructions. The tumors were measured using calipers every 3 days and the equation for volume was $\mathrm{V}=\mathrm{LxWxHx}(\pi / 6)$. At the study end or if the tumors reached $1.5 \mathrm{cc}$ the tumors were removed and measured.

Quail toxicity assay. All experiments were conducted in accordance with Children's Cancer Therapy Development Institute policies and all relevant guidelines. Cediranib (Cat\# S1017, Selleck Chemicals LLC, Houston, TX) and erlotinib (Cat\# S7786) were purchased from Selleck Chemicals and reconstituted in dimethyl sulfoxide (DMSO) following the manufacturers recommendations and diluted to $10 \mathrm{mM}$ stock concentration.

Fertilized Japanese quail eggs were incubated and plated as described previously. Quail were allowed to grow ex ovo in six-well plates until the quail had passed the patterning phase (e8 based on plating date). Each experimental arm was assigned $n=4$ viable quail and treated with one of four experimental conditions: vehicle, cediranib, erlotinib, and cediranib + erlotinib. Dosages provided to quail were based on maximum clinicallyachievable serum concentrations $\left(\mathrm{C}_{\max }\right)$ in human patients, specifically $42 \mathrm{ng} / \mathrm{mL}$ for cediranib ${ }^{13}$ and $1.3 \mu \mathrm{g} / \mathrm{mL}$ for erlotinib ${ }^{14}$. Stock concentrations were diluted in phosphate-buffered saline (PBS) to the respective target concentrations and to a final volume of $25 \mu \mathrm{L}$ per agent. DMSO for vehicle was set at the DMSO volume used in the cediranib + erlotinib combination (8.4 $\mu \mathrm{L}$ DMSO).

Vehicle and diluted agents were subsequently applied dropwise to the quail chorioallantoic membrane. Quail were photographed at $0 \mathrm{~h}, 24 \mathrm{~h}$, and $48 \mathrm{~h}$. Remaining viable quail ( $\mathrm{n}=4$ vehicle, $\mathrm{n}=3$ cediranib, $\mathrm{n}=3$ erlotinib, $\mathrm{n}=4$ combination) were sacrificed $48 \mathrm{~h}$ after dosing and fixed in $10 \%$ formalin for $24 \mathrm{~h}$. Fixed quail were transported to the OHSU Histology core, paraffin embedded, sectioned in coronal orientation, and stained with hematoxylin and eosin. Stained images were analyzed by pathologist co-author A.M. for kidney and liver histopathology looking for signs of normal versus abnormal development (Fig. 5).

Sequencing of samples. Each cell line was grown to $80 \%$ confluency, trypsinized, and snap frozen. RNA was extracted and sequenced by Beijing Genomics Institute (BGI, San Jose, CA). The quality of RNA prior to extraction was adequate for each cell line (DV $<200 \%)$. HiSeq 4000 was used for paired-end sequencing with 40 million reads for RNA. Raw FASTQ sequencing files were run through our in-house computational pipeline.

Statistical analysis. For the murine in vivo xenograft study survival analysis, the tumor endpoint volumes for time-to-event (TTE) analysis were set at $0.75 \mathrm{cc}$ and were collected to $1.5 \mathrm{cc}$. TTE was defined in days by selecting the day in which the tumor volume equaled or surpassed $0.75 \mathrm{cc}$. Animals that did not reach endpoint volume were assigned a TTE of 21 days for the HB243 analysis and a TTE of 12 days for the G2 analysis. The Kaplan-Meier survival plot represents the percentage of animals surviving at different time points during the study. These percentages were generated from the TTE data using GraphPad Prism 9.0 software (Graphpad Software, San Diego, CA, https://www.graphpad.com/scientific-software/prism/). Survival curve comparisons were analyzed using the Mantel-Cox and Gehan-Breslow-Wilcox tests (95\% CI) through Graph Pad Prism 9.0 software. For the quail xenograft assay, significance was determined by an unpaired two-tailed t-test with Welch's 
correction and a p-value less than 0.05 was considered statistically significant. Error bars represent \pm standard error of the mean (SEM).

Received: 5 April 2021; Accepted: 10 November 2021

Published online: 02 December 2021

\title{
References
}

1. Abraham, J. et al. Evasion mechanisms to Igf1r inhibition in rhabdomyosarcoma. Mol. Cancer Ther. 10, 697-707 (2011).

2. Aslam, M. I. et al. PDGFRbeta reverses EphB4 signaling in alveolar rhabdomyosarcoma. Proc. Natl. Acad. Sci. U.S.A. 111, 63836388 (2014).

3. Lokman, N. A., Elder, A. S., Ricciardelli, C. \& Oehler, M. K. Chick chorioallantoic membrane (CAM) assay as an in vivo model to study the effect of newly identified molecules on ovarian cancer invasion and metastasis. Int. J. Mol. Sci. 13, 9959-9970 (2012).

4. Subauste, M. C. et al. Evaluation of metastatic and angiogenic potentials of human colon carcinoma cells in chick embryo model systems. Clin. Exp. Metastasis 26, 1033-1047 (2009).

5. Handler, A. H., Geiser, C. F. \& Farber, S. Studies on mortality in chick embryos resulting from implantation of whole blood and blood fractions from patients and animals with neoplastic disease. Proc. Natl. Acad. Sci. U.S.A. 48, 1549-1573 (1962).

6. Ainsworth, S. J., Stanley, R. L. \& Evans, D. J. Developmental stages of the Japanese quail. J. Anat. 216, 3-15 (2010).

7. Deryugina, E. I. \& Quigley, J. P. Chick embryo chorioallantoic membrane model systems to study and visualize human tumor cell metastasis. Histochem. Cell Biol. 130, 1119-1130 (2008).

8. Atsushi Kato, D. M. et al. Culture system for bobwhite quail embryos from the blastoderm stage to hatching.pdf. J. Poultry Sci. 2, 155-158 (2013).

9. Vargas, A., Zeisser-Labouebe, M., Lange, N., Gurny, R. \& Delie, F. The chick embryo and its chorioallantoic membrane (CAM) for the in vivo evaluation of drug delivery systems. Adv. Drug Deliv. Rev. 59, 1162-1176 (2007).

10. Kats, D. et al. Volasertib preclinical activity in high-risk hepatoblastoma. Oncotarget 10, 6403-6417 (2019).

11. Lopez-Terrada, D., Cheung, S. W., Finegold, M. J. \& Knowles, B. B. Hep G2 is a hepatoblastoma-derived cell line. Hum. Pathol. 40, 1512-1515 (2009).

12. Stebbing, J. et al. Patient-derived xenografts for individualized care in advanced sarcoma. Cancer 120, 2006-2015 (2014).

13. Fox, E. et al. A phase 1 trial and pharmacokinetic study of cediranib, an orally bioavailable pan-vascular endothelial growth factor receptor inhibitor, in children and adolescents with refractory solid tumors. J. Clin. Oncol. 28, 5174-5181 (2010).

14. Broniscer, A. et al. Phase I and pharmacokinetic studies of erlotinib administered concurrently with radiotherapy for children, adolescents, and young adults with high-grade glioma. Clin. Cancer Res. 15, 701-707 (2009).

\section{Acknowledgements}

Funding for this work was supported by the Macy Easom Cancer Research Foundation, as well as The Foundation for Addie's Research, Owls for Avery Foundation, Rutledge Foundation, the Super Sam Foundation, the Go4TheGoal Foundation and the Sam Day Foundation. Histology for this work was done by the OHSU Histopathology Shared Resource.

\section{Author contributions}

Overall conception and design: C.K. Analysis and interpretation of experimental data: C.K., S.V.R., L.H.P., A.M., S.R. Development of experimental methodology: C.K., S.V.R., N.E.B. Provision of experimental reagents: S.C., S.V.R. Acquisition of experimental data: S.V.R., N.E.B., S.R. Writing, review and/or revision: C.K., S.V.R., S.R., S.C. Study supervision: C.K.

\section{Competing interests}

The authors declare no competing interests.

\section{Additional information}

Supplementary Information The online version contains supplementary material available at https://doi.org/ 10.1038/s41598-021-02509-3.

Correspondence and requests for materials should be addressed to C.K.

Reprints and permissions information is available at www.nature.com/reprints.

Publisher's note Springer Nature remains neutral with regard to jurisdictional claims in published maps and institutional affiliations.

\begin{abstract}
(c) (1) Open Access This article is licensed under a Creative Commons Attribution 4.0 International cc) License, which permits use, sharing, adaptation, distribution and reproduction in any medium or format, as long as you give appropriate credit to the original author(s) and the source, provide a link to the Creative Commons licence, and indicate if changes were made. The images or other third party material in this article are included in the article's Creative Commons licence, unless indicated otherwise in a credit line to the material. If material is not included in the article's Creative Commons licence and your intended use is not permitted by statutory regulation or exceeds the permitted use, you will need to obtain permission directly from the copyright holder. To view a copy of this licence, visit http://creativecommons.org/licenses/by/4.0/.
\end{abstract}

(C) The Author(s) 2021 